\title{
Treatment of nephrotic syndrome with adrenocorticotropic hormone (ACTH) gel
}

This article was published in the following Dove Press journal:

Drug Design, Development and Therapy

I I March 20II

Number of times this article has been viewed

\author{
Andrew S Bomback' \\ James A Tumlin ${ }^{2}$ \\ Joel Baranski ${ }^{3}$ \\ James E Bourdeau ${ }^{4}$ \\ Anatole Besarab 5 \\ Alice S Appel' \\ Jai Radhakrishnan' \\ Gerald B Appel'
}

'Department of Medicine, Division of Nephrology, Columbia University College of Physicians and Surgeons, New York, NY, USA; ${ }^{2}$ Department of Internal Medicine, Division of Nephrology, University of Tennessee College of Medicine in Chattanooga, Chattanooga, TN, USA; ${ }^{3}$ Balboa Nephrology Medical Group, San Diego, CA, USA; ${ }^{4} \mathrm{Nephrology}$ Specialists of Oklahoma, Tulsa, OK, USA; ${ }^{5}$ Department of Medicine, Division of Nephrology and Hypertension, Henry Ford Health System, Detroit, MI, USA
Correspondence: Andrew S Bomback Columbia University College of Physicians and Surgeons, Division of Nephrology, 622 West 168th Street, PH 4-I24,

New York, NY 10032, USA

$\mathrm{Tel}+$ I 2/2-305-0320

Fax + I 212-305-6678

Email asb68@columbia.edu
Purpose: A synthetic adrenocorticotropin (ACTH) analog has shown efficacy in Europe as primary and secondary therapy for nephrotic syndrome, but there is no published experience using the natural, highly purified ACTH gel formulation, available in the United States, for nephrotic syndrome. We therefore investigated the use of ACTH gel for nephrotic syndrome in the United States.

Patients and methods: Twenty-one patients with nephrotic syndrome treated with ACTH gel outside of research settings in the United States, with initiation of therapy by December 31 , 2009, allowing a minimum 6 months follow-up. We defined complete remission as stable renal function with proteinuria falling to $<500 \mathrm{mg} /$ day, and partial remission as stable renal function with $>50 \%$ reduction in proteinuria from 500 to $3500 \mathrm{mg}$ /day.

Results: Twenty-one patients with nephrotic syndrome were treated: 11 with idiopathic membranous nephropathy (iMN), 4 with membranoproliferative glomerulonephritis (MPGN), 1 with focal segmental glomerulosclerosis (FSGS), 1 with minimal change disease (MCD), 1 with immunoglobulin A (IgA) nephropathy, 1 with class V systemic lupus erythematosus (SLE) glomerulonephritis, 1 with monoclonal diffuse proliferative glomerulonephritis, and 1 with unbiopsied nephrotic syndrome. ACTH was used as primary therapy for 3 patients; the remaining patients had previously failed a mean 2.3 immunosuppressive regimens. Eleven patients achieved a complete or partial remission, with 4 (19\%) in complete remission. Of the 11 patients who achieved remission, 9 had iMN, 1 had FSGS, and 1 had IgA nephropathy. Of the 11 patients with iMN, $3(27 \%)$ achieved complete remission and $6(55 \%)$ achieved partial remission despite having previously failed a mean 2.4 therapies. Five patients reported steroid-like adverse effects, but there were no severe infections. The limitations were retrospective data analysis with short-term follow-up.

Conclusion: ACTH gel may be a viable treatment option for resistant nephrotic syndrome due to membranous nephropathy. Short-term data suggest that remission rates may approach $80 \%$.

Keywords: nephrotic syndrome, membranous nephropathy, chronic kidney disease

\section{Introduction}

Patients with nephrotic syndrome often require immunosuppression to achieve remission, yet many patients either relapse after remission or are resistant to therapy. For example, while up to $90 \%$ of adults with minimal change disease (MCD) will respond to initial therapy with prednisone, approximately one-third of these same patients will relapse within 6 months and require further immunosuppression. ${ }^{1,2}$ With diseases such as idiopathic membranous nephropathy (iMN) and focal segmental glomerulosclerosis (FSGS), for which first-line therapies produce substantially lower 
response rates than for $\mathrm{MCD}$, physicians are often compelled to use second-, third-, and even fourth-line therapies to achieve remission. ${ }^{3-8}$

In several European studies, tetracosactide, a synthetic adrenocorticotropic hormone (ACTH) analog, has shown efficacy as primary and secondary therapy for nephrotic syndrome. The initial reports came in a case series of patients with various etiologies of nephrotic range proteinuria, including MCD, iMN, FSGS, and membranoproliferative glomerulonephritis (MPGN). ${ }^{9}$ Subsequently, a randomized, controlled study by Ponticelli et al reported similar remission rates in patients with iMN randomized to synthetic ACTH or to therapy with alternating months of steroids and cyclophosphamide. ${ }^{10}$ These reports have generated renewed interest in using ACTH as treatment for nephrotic syndrome, particularly in patients who are resistant to conventional therapies. Synthetic ACTH is not currently available for use in the United States, but a natural, highly purified ACTH gel formulation (H.P. Acthar ${ }^{\circledR}$ Gel [repository corticotropin injection], Questcor Pharmaceuticals, Inc, Union City, CA, USA; abbreviated ACTH gel) is both available and approved for use in nephrotic syndrome.

To date, however, there is no modern published experience on using ACTH gel in nephrotic patients. We therefore explored the initial use of ACTH gel for nephrotic syndrome in nonresearch settings (ie, by prescription), collecting data from treating nephrologists of all known patients in the United States whose treatment with this agent was initiated by the end of 2009. We describe their clinical course before and after treatment with ACTH gel.

\section{Material and methods}

In this retrospective case series, we evaluated all known cases of idiopathic, nondiabetic nephrotic syndrome treated with ACTH gel outside of research settings (ie, by prescription) with initiation of therapy by December 31, 2009, allowing a minimum of 6 months' follow-up. Questcor Pharmaceuticals, the drug's manufacturer, provided contact information for nephrologists whose patients had ACTH gel prescriptions filled during this time period. Treating nephrologists were asked to provide data on patients' demographics, diagnoses, previous immunosuppressive regimens, indications for ACTH gel therapy, dosing of ACTH gel, clinical response to therapy (creatinine, proteinuria, serum albumin, cholesterol), and adverse events from initiation of therapy to most recent clinical encounter.

We defined complete remission as stable or improved renal function (estimated glomerular filtration rate, GFR
[eGFR], based on serum creatinine) with final proteinuria falling to $<500 \mathrm{mg} /$ day; partial remission as stable or improved renal function with $\geq 50 \%$ reduction in proteinuria and final proteinuria 500 to $3500 \mathrm{mg} /$ day; and limited response as stable or improved renal function with $\geq 50 \%$ reduction in proteinuria but final proteinuria $>3500 \mathrm{mg} /$ day. Failure to meet the above criteria was classified as no response. Given the small number of patients and the observational nature of this study, the data are presented descriptively, and no formal statistical analyses were performed.

\section{Results}

\section{Patient characteristics}

In the United States, 25 patients with nondiabetic nephrotic syndrome began treatment with ACTH gel before December 31, 2009. Data were not available for 4 patients with unknown diagnoses. Full data were available for 21 patients with the following diagnoses (Table 1): iMN $(n=11), \operatorname{MPGN}(n=4), \operatorname{FSGS}(n=1), \operatorname{MCD}(n=1)$, immunoglobulin A $(\operatorname{Ig} \mathrm{A})$ nephropathy $(\mathrm{n}=1)$, class $\mathrm{V}$ systemic lupus erythematosus (SLE) glomerulonephritis $(\mathrm{n}=1)$, monoclonal diffuse proliferative glomerulonephritis $(\mathrm{n}=1)$, and unbiopsied nephrotic syndrome $(n=1)$.

ACTH gel was used as primary therapy for only 3 patients, one each with diagnoses of iMN, IgA nephropathy, and nonbiopsied nephrotic syndrome. The remaining 18 patients had failed a mean 2.3 immunosuppressive regimens prior to ACTH gel therapy, of which 9 patients had failed at least 3 prior therapies. Nine of 21 patients were female, all but 3 patients were white, and there was a wide range of ages ( 24 to 81 years). Most patients had impaired baseline renal function (eGFR range from 0.2 to $>1.0 \mathrm{~mL} / \mathrm{s} / 1.73 \mathrm{~m}^{2}\left[11\right.$ to $\left.>60 \mathrm{~mL} / \mathrm{min} / 1.73 \mathrm{~m}^{2}\right]$ ) with 12 patients demonstrating stage 4 or 5 chronic kidney disease $\left(e G F R \leq 0.5 \mathrm{~mL} / \mathrm{s} / 1.73 \mathrm{~m}^{2}\left[\leq 30 \mathrm{~mL} / \mathrm{min} / 1.73 \mathrm{~m}^{2}\right]\right)$. Pretreatment proteinuria ranged from $1340 \mathrm{mg} /$ day to $18,553 \mathrm{mg}$ /day; only 3 patients had proteinuria $<4000 \mathrm{mg}$ /day, while 11 had proteinuria $>8000 \mathrm{mg} /$ day (Table 2). The 3 patients whose baseline proteinuria was in subnephrotic range exhibited other signs of nephrotic syndrome (hypoalbuminemia, hyperlipidemia, and edema). Mean pretreatment albumin (data available for 19 patients) was $29 \mathrm{~g} / \mathrm{L}$ $(2.9 \mathrm{~g} / \mathrm{dL})$, and mean pretreatment total cholesterol was $6.10 \mathrm{mmol} / \mathrm{L}$ (236 mg/dL) (data available for 12 patients).

The most common treatment regimen was 80 units subcutaneously (SC) twice weekly for 6 months, which was used in 13 of 21 patients (Table 2). All but 6 were treated for a minimum 
Table I Characteristics of patients in the United States treated with ACTH gel for nephrotic syndrome through December 3I, 2009

\begin{tabular}{|c|c|c|c|c|c|c|c|}
\hline Patient & Age & Gender & $\begin{array}{l}\text { Racel } \\
\text { ethnicity }\end{array}$ & Diagnosis & $\begin{array}{l}\text { Previous } \\
\text { immunosuppression }\end{array}$ & $\begin{array}{l}\text { eGFR } \\
\left(\mathrm{mL} / \mathrm{min} / 1.73 \mathrm{~m}^{2}\right)\end{array}$ & $\begin{array}{l}\text { Proteinuria } \\
\text { (mg/day) }\end{array}$ \\
\hline 1 & 59 & Male & White & $\mathrm{iMN}$ & MMF, CNI & $>60$ & 4851 \\
\hline 2 & 77 & Male & Hispanic & iMN & MMF, CNI & 21 & 6749 \\
\hline 3 & 58 & Male & White & $\mathrm{iMN}$ & Steroids + CTX, CNI, MMF & 58 & 4598 \\
\hline 4 & 55 & Male & White & $\mathrm{iMN}$ & Steroids + CTX, CNI, MMF & 57 & 8153 \\
\hline 5 & 27 & Male & White & iMN & Steroids, MMF, CNI & 30 & 9000 \\
\hline 6 & 24 & Female & White & $\mathrm{iMN}$ & None & $>60$ & 8900 \\
\hline 7 & 75 & Female & White & $\mathrm{iMN}$ & Steroids + CTX & $>60$ & 3469 \\
\hline 8 & 49 & Male & White & iMN & Steroids, CNI & 25 & 9150 \\
\hline 9 & 46 & Male & White & $\mathrm{iMN}$ & Steroids + CTX & 20 & $\mid 1,911$ \\
\hline 10 & 53 & Male & White & iMN & Steroids, Steroids + CTX & $>60$ & 5700 \\
\hline 11 & 70 & Female & White & $\mathrm{iMN}$ & Steroids, CNI, CTX & 40 & 2625 \\
\hline 12 & 81 & Male & White & MPGN & Steroids, MMF, rituximab & 25 & 13,073 \\
\hline 13 & 28 & Female & White & MPGN & Steroids, MMF, rituximab & 23 & 5500 \\
\hline 14 & 53 & Female & White & MPGN & Steroids, MMF & II & 12,398 \\
\hline 15 & 47 & Female & White & MPGN & MMF & 21 & 10,244 \\
\hline 16 & 57 & Female & White & MCD & Steroids, MMF, CNI & 15 & 18,553 \\
\hline 17 & 63 & Female & Hispanic & FSGS & Steroids, MMF, CNI & 33 & 10275 \\
\hline 18 & 75 & Male & Black & IgA nephropathy & None & 22 & 4952 \\
\hline 19 & 32 & Female & White & SLE class $V$ & Steroids + MMF + CNI & $>60$ & 1340 \\
\hline 20 & 36 & Male & White & Monoclonal DPGN & Steroids, rituximab, MMF, CNI & 19 & 8560 \\
\hline 21 & 74 & Male & White & $N S^{a}$ & None & 12 & 5805 \\
\hline
\end{tabular}

Notes: Conversion factor for eGFR: $\mathrm{mL} / \mathrm{min} / 1.73 \mathrm{~m}^{2}$ to $\mathrm{mL} / \mathrm{s} / 1.73 \mathrm{~m}^{2}, \times 0.01667$; a Patient with nephrotic syndrome who did not undergo biopsy for specific diagnosis. Abbreviations: CNI, calcineurin inhibitor; CTX, cyclophosphamide; DPGN, diffuse proliferative glomerulonephritis; eGFR, estimated glomerular filtration rate; FSGS, focal segmental glomerulosclerosis; IgA, immunoglobulin A; iMN, idiopathic membranous nephropathy; MCD, minimal change disease; MMF, mycophenolate mofetil; MPGN, membranoproliferative glomerulonephritis; NS, nephrotic syndrome; SLE, systemic lupus erythematosus.

of 6 months. Five patients treated for fewer than 6 months were taken off therapy as a result of clear lack of response; 1 patient discontinued therapy due to weight gain but had achieved a temporary response while on therapy. The longest treatment duration was 14 months, of which the last 2 months included a taper of the dose from 40 units twice weekly to 40 units once weekly. Most patients stopped treatment without a taper, however, and without obvious rebound in proteinuria.

\section{Outcomes}

Overall, 11 of 21 patients (52\%) achieved a complete or partial remission, with $4(19 \%)$ in complete remission (Table 2). One patient achieved a limited response, while 9 patients had no overall response to therapy. The patient with class V SLE glomerulonephritis transiently responded to therapy (proteinuria declining to $<500 \mathrm{mg} /$ day) but relapsed as soon as therapy was stopped, thus not meeting criteria for remission. Of the 11 patients who achieved complete or partial remission, 9 had iMN, 1 had FSGS, and 1 had IgA nephropathy. Of the 11 patients with iMN (Figure 1), 3 achieved complete remission and 6 achieved partial remission despite having previously failed a mean 2.4 therapies. Of the 10 patients with nephrotic syndrome diagnoses other than iMN (Figure 2), 1 patient with IgA nephropathy achieved complete remission,
1 patient with FSGS achieved partial remission, and 1 patient with MPGN had a limited response to therapy.

Follow-up time for patients ranged from 6 to 14 months. Five patients reported steroidlike adverse effects with therapy: 2 patients had impaired blood glucose control, 2 patients had significant weight gain, and 1 patient showed evidence of accelerated bone loss on bone densitometry not observed prior to ACTH gel therapy (Table 3). In addition, 1 patient with monoclonal diffuse proliferative glomerulonephritis developed acute renal failure within 1 month of starting therapy, with creatinine rising from $327.1 \mu \mathrm{mol} / \mathrm{L}(3.7 \mathrm{mg} / \mathrm{dL})$ to $751.4 \mu \mathrm{mol} / \mathrm{L}(8.5 \mathrm{mg} / \mathrm{dL})$. The drug was stopped along with the patient's diuretics and angiotensin-converting enzyme inhibitor; after intravenous fluid hydration, the creatinine returned to $353.6 \mu \mathrm{mol} / \mathrm{L}$ $(4.0 \mathrm{mg} / \mathrm{dL})$. Six months after starting ACTH gel, the patient's creatinine ranged between 442.0 and $530.4 \mu \mathrm{mol} / \mathrm{L}$ (5.0 and $6.0 \mathrm{mg} / \mathrm{dL}$ ), with transplant evaluation underway. The other 15 patients reported no adverse events. No severe infections were reported in the entire cohort.

\section{Discussion}

We present here a retrospective case series evaluating the initial experience of 21 patients in the United States treated 
Table 2 Dosing, duration, and outcomes of ACTH gel therapy for nephrotic syndrome

\begin{tabular}{|c|c|c|c|c|c|c|c|}
\hline Patient & Diagnosis & $\begin{array}{l}\text { ACTH } \\
\text { gel dose (units) }\end{array}$ & $\begin{array}{l}\text { Duration of ACTH } \\
\text { therapy }\end{array}$ & $\begin{array}{l}\text { Follow-up } \\
\text { time }\end{array}$ & $\begin{array}{l}\text { Pre-ACTH } \\
\text { proteinuria } \\
\text { (mg/day) }\end{array}$ & $\begin{array}{l}\text { Last proteinuria } \\
\text { (mg/day) }\end{array}$ & Outcome \\
\hline $\mathrm{I}$ & iMN & 80 SC twice weekly & 6 months & 8 months & 4851 & 400 & Complete remission \\
\hline 2 & iMN & 80 SC twice weekly & 6 months & 6 months & 6749 & 1540 & Partial remission \\
\hline 3 & iMN & 80 SC twice weekly & 6 months & 6 months & 4598 & 1242 & Partial remission \\
\hline 4 & iMN & 80 SC twice weekly & 6 months & 6 months & 8153 & 1935 & Partial remission \\
\hline 5 & iMN & 80 SC twice weekly & 6 months & 6 months & 9000 & 3000 & Partial remission \\
\hline 6 & iMN & 80 SC twice weekly & 5 months & 12 months & 8900 & 6000 & No response \\
\hline 7 & iMN & 40 SC twice weekly & 12 months & 14 months & 3469 & 34 & Complete remission \\
\hline 8 & iMN & $80 \mathrm{SC}$ q $72 \mathrm{hrs}$ & II months & II months & 9150 & 2948 & Partial remission \\
\hline 9 & iMN & 40 SC thrice weekly & 6 months & 7 months & 11911 & 13,338 & No response \\
\hline 10 & iMN & 40 SC thrice weekly & 6 months & 6 months & 5700 & 694 & Partial remission \\
\hline II & $\mathrm{iMN}$ & 80 SC twice weekly & 12 months & 13 months & 2625 & 240 & Complete remission \\
\hline 12 & MPGN & 80 SC twice weekly & 4.5 months & 8 months & 13073 & $374 I$ & No response ${ }^{a}$ \\
\hline 13 & MPGN & 80 SC twice weekly & 4 months & 6 months & 5500 & 4825 & No response \\
\hline 14 & MPGN & 40 SC thrice weekly & 6 months & 6 months & 12398 & 4560 & Limited response \\
\hline 15 & MPGN & 80 SC twice weekly & 6 months & 6 months & 10244 & 3878 & No response ${ }^{\mathrm{a}}$ \\
\hline 16 & MCD & 80 SC twice weekly & 4 months & 8 months & 18553 & 18,557 & No response \\
\hline 17 & FSGS & 80 SC twice weekly & 6 months & 6 months & 10275 & 2970 & Partial remission \\
\hline 18 & $\begin{array}{l}\text { IgA } \\
\text { nephropathy }\end{array}$ & $40 \mathrm{SC}$ q $72 \mathrm{hrs}$ & 8 months & 8 months & 4952 & 42 & Complete remission \\
\hline 19 & SLE class $V$ & 40 SC thrice weekly & 5 months & 8 months & 1340 & 2290 & No response ${ }^{b}$ \\
\hline 20 & $\begin{array}{l}\text { Monoclonal } \\
\text { DPGN }\end{array}$ & 80 SC twice weekly & I month & 6 months & 8560 & 8500 & No response \\
\hline 21 & NS & 40 SC q 72 hrs & 6 months & 9 months & 5805 & 8708 & No response \\
\hline
\end{tabular}

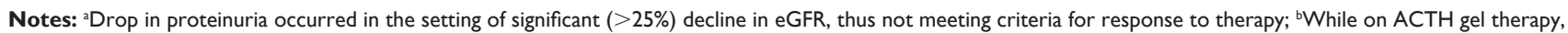
proteinuria dropped from $1340 \mathrm{mg} /$ day to $420 \mathrm{mg} /$ day; after patient discontinued therapy due to concerns of weight gain, her proteinuria rebounded to $2290 \mathrm{mg} /$ day.

Abbreviations: ACTH, adrenocorticotropin; DPGN, diffuse proliferative glomerulonephritis; FSGS, focal segmental glomerulosclerosis; IgA, immunoglobulin A; iMN, idiopathic membranous nephropathy; MCD, minimal change disease; MPGN, membranoproliferative glomerulonephritis; NS, nephrotic syndrome without biopsy; SC, subcutaneous; SLE, systemic lupus erythematosus.

with ACTH gel for nephrotic syndrome. The majority of these patients received ACTH as second-, third-, or fourth-line therapy for resistant nephrotic syndrome. Idiopathic membranous nephropathy was the leading diagnosis among these patients and also showed the greatest benefit of therapy, with 9 of 11 patients $(82 \%)$ achieving either complete or

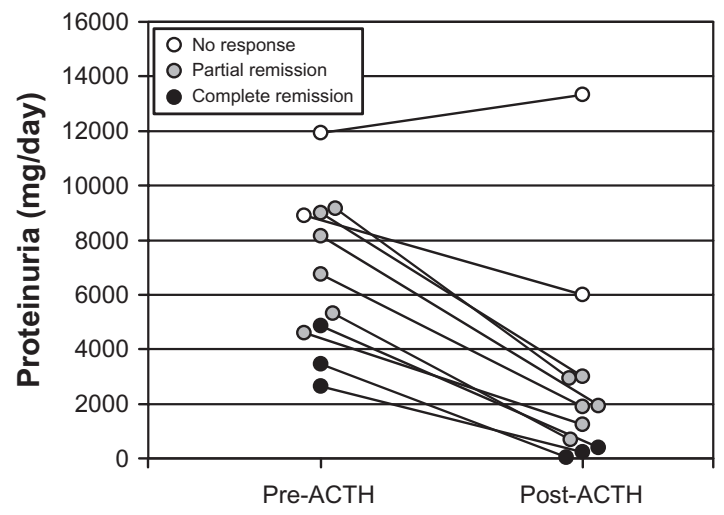

Figure I Changes in proteinuria with ACTH gel therapy in II patients with nephrotic syndrome due to membranous nephropathy.

Abbreviation: ACTH, adrenocorticotropin. partial remission. Adverse events associated with therapy were relatively minor and included no significant infections. Although 3 patients with other etiologies of nephrotic syndromedemonstrated response to therapy, the early clinical data suggest that the drug's greatest potential benefit may be in treating refractory iMN.

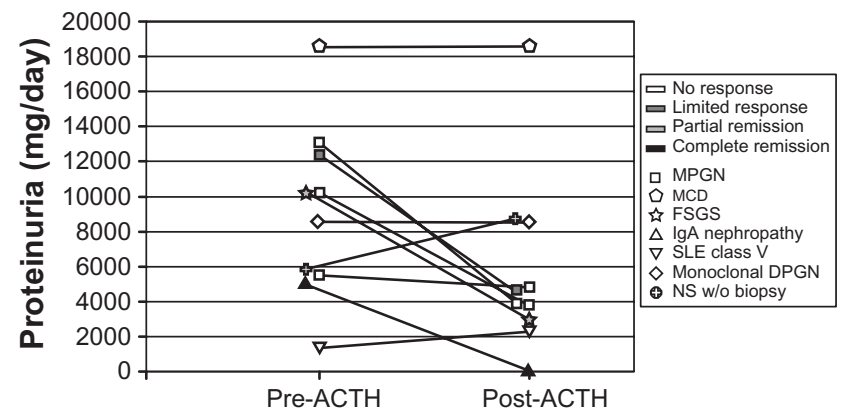

Figure 2 Changes in proteinuria with ACTH gel therapy in 10 patients with nephrotic syndrome due to etiologies other than membranous nephropathy. Abbreviations: ACTH, adrenocorticotropin; FSGS, focal segmental glomerulosclerosis; IgA, immunoglobulin A; MCD, minimal change disease; MPGN, membranoproliferative glomerulonephritis; NS, nephrotic syndrome without biopsy; SLE, systemic lupus erythematosus 
Table 3 Reported adverse events of ACTH gel therapy

\begin{tabular}{lllll}
\hline Patient & Diagnosis & ACTH gel dose (units) & Duration of ACTH therapy & Adverse events \\
\hline 1 & iMN & $80 \mathrm{SC}$ twice weekly & 6 months & None \\
2 & iMN & $80 \mathrm{SC}$ twice weekly & 6 months & None \\
3 & iMN & $80 \mathrm{SC}$ twice weekly & 6 months & None \\
4 & iMN & $80 \mathrm{SC}$ twice weekly & 6 months & Hyperglycemia \\
5 & iMN & $80 \mathrm{SC}$ twice weekly & 6 months & None \\
6 & iMN & $80 \mathrm{SC}$ twice weekly & 5 months & Weight gain \\
7 & iMN & $40 \mathrm{SC}$ twice weekly & 12 months & Bone demineralization \\
8 & iMN & $80 \mathrm{SC}$ q 72 h & None \\
9 & iMN & $40 \mathrm{SC}$ thrice weekly & 6 months & None \\
10 & iMN & $40 \mathrm{SC}$ thrice weekly & 6 months & None \\
11 & iMN & $80 \mathrm{SC}$ twice weekly & 12 months & Hyperglycemia \\
12 & MPGN & $80 \mathrm{SC}$ twice weekly & 4.5 months & None \\
13 & MPGN & $80 \mathrm{SC}$ twice weekly & 4 months & None \\
14 & MPGN & $40 \mathrm{SC}$ thrice weekly & 6 months & None \\
15 & MPGN & $80 \mathrm{SC}$ twice weekly & 6 months & None \\
16 & MCD & $80 \mathrm{SC}$ twice weekly & 4 months & None \\
17 & FSGS & $80 \mathrm{SC}$ twice weekly & 6 months & None \\
18 & IgA nephropathy & $40 \mathrm{SC}$ q $72 \mathrm{~h}$ & 8 months & None \\
19 & SLE class V & $40 \mathrm{SC}$ thrice weekly & 5 months & Weight gain \\
20 & Monoclonal DPGN & $80 \mathrm{SC}$ twice weekly & 1 month & Acute renal failure \\
21 & NS & $40 \mathrm{SC}$ q 72 h & 6 months & None \\
\hline
\end{tabular}

Note: a Creatinine returned to baseline after IV hydration alongside cessation of ACTH gel, diuretics, and angiotensin converting enzyme-inhibitor.

Abbreviations: ACTH, adrenocorticotropin; DPGN, diffuse proliferative glomerulonephritis; FSGS, focal segmental glomerulosclerosis; IgA, immunoglobulin A; MN, idiopathic membranous nephropathy; MCD, minimal change disease; MPGN, membranoproliferative glomerulonephritis; NS, nephrotic syndrome without biopsy; SC, subcutaneous; SLE, systemic lupus erythematosus.

Our observations are concordant with previously published reports from Europe using a synthetic ACTH analog for nephrotic syndrome due to iMN. Berg and Arnadottir, in a seminal paper from 2004, reported the results of ACTH treatment in 23 cases of nephrotic syndrome of various etiologies, including 10 cases of iMN. ${ }^{9}$ Half of these iMN patients had previously been treated with at least 1 prior immunosuppressive therapy, and all 10 achieved sustained remission of proteinuria. A more recent series from Germany reported the results of 8 months of synthetic ACTH therapy for 4 patients with iMN refractory to prior therapies of steroids, cyclosporine, cyclophosphamide, mycophenolate mofetil, or azathioprine. ${ }^{11}$ Within the first year, 2 had achieved complete remission, and 2 had achieved partial remission. The most convincing data, however, come from the randomized trial by Ponticelli et al, in which 16 patients with iMN received steroids alternating with a cytotoxic drug for 6 months versus 16 patients with iMN treated with synthetic ACTH for 1 year. ${ }^{10}$ Most of these patients were on renin angiotensin system blocking drugs, but none had previously received immunosuppression. After a median follow-up of 24 months, there were 4 complete and 8 partial remissions in the steroid/cytotoxic therapy group versus 8 complete and 6 partial remissions in the ACTH group.
The results presented here, on the other hand, do not wholly agree with the successful experiences of Berg and Arnadottir in treating nephrotic syndromes other than iMN. ${ }^{9}$ Thirteen of the 23 cases presented in their series were nephrotic syndrome diagnoses other than iMN. Only 1 patient with MPGN did not respond to synthetic ACTH. The remaining 12 patients - with diagnoses of MPGN, MCD, FSGS, diabetic nephropathy, and hereditary nephritis - all experienced at least a $50 \%$ reduction in proteinuria during synthetic ACTH therapy, and 8 patients achieved sustained complete remission (proteinuria $<500 \mathrm{mg}$ /day). In this case series, US patients treated with ACTH gel for diagnoses other than iMN did not fare as well: for example, only 1 of 4 MPGN patients demonstrated a limited response. In part, this may be due to these patients having worse baseline renal function and having failed more therapies than those reported by Berg and Arnadottir. Notably, 11 of the 21 patients reported here had advanced renal insufficiency $\left(\right.$ GFR $\left.<0.5 \mathrm{~mL} / \mathrm{s} / 1.73 \mathrm{~m}^{2}\left[30 \mathrm{~mL} / \mathrm{min} / 1.73 \mathrm{~m}^{2}\right]\right)$ when prescribed ACTH. Eight of these 11 patients were diagnosed with diseases other than $\mathrm{MN}$, and their response rate to ACTH was generally poor. The lack of response exhibited by these patients may reflect the degree of renal failure more than the specific diagnosis itself. Theoretically, the difference in response could also be explained by inherent 
differences between the synthetic and natural formulations of $\mathrm{ACTH}$.

Although encouraging, the initial results of ACTH gel therapy in the United States must be interpreted cautiously in light of the limitations of an observational series such as this. These patients were not randomized to therapy, and there is no comparison or control group against which to interpret these results. In some respects, however, the patients may serve as their own controls, having failed on average between 2 and 3 prior immunosuppressive regimens. We did not have detailed data on duration of time between prior immunosuppressive therapies and initiation of ACTH gel; conceivably, if this duration was short, some of the response could be attributed to a delay in response to prior agents. In addition, the data presented here reflect only short-term follow-up, most patients having less than 1 year of follow-up at the time of this report. Given that 1 of the patients presented here has relapsed off therapy, the follow-up period is likely too short to label the remissions as sustained. In previous reports of synthetic ACTH therapy, some patients have relapsed but responded to second courses of ACTH with good results. ${ }^{9,11}$ The short duration of follow-up may also understate adverse events in this cohort, which thus far have been mild and mostly steroidlike in presentation. Finally, our report does not address cost analysis of the various agents available to treat the nephrotic syndrome.

These data also do not provide any further understanding of the mechanism of action by which ACTH ameliorates proteinuria in the nephrotic syndrome. Speculatively, its better performance in iMN than other causes of nephrotic syndrome might point to a target of action; eg, antibodies against the phospholipase A2 receptor. ${ }^{12}$ However, a recent study using rats with passive Heymann nephritis, an animal model of membranous nephropathy, proposes that ACTH may work at the melanocortin receptor MC1R in podocytes to reduce proteinuria, improve glomerular morphology, and reduce oxidative stress. ${ }^{13}$ This finding suggests that ACTH should be equally, if not more, effective in podocytopathies such as MCD and FSGS than in iMN. As only 2 of our patients had MCD or FSGS, further study of ACTH gel in these conditions is clearly warranted.

\section{Conclusion}

In conclusion, we present a case series on the initial experience in the United States of using ACTH gel as treatment of nephrotic syndrome. Overall, 11 of 21 patients - including 10 of 18 patients who could be classified as treatment resistant - achieved either complete or partial remission with at least 6 months of follow-up. Further, 9 of 11 patients with refractory nephrotic syndrome due to iMN achieved remission despite having previously failed at least 2 prior immunosuppressive regimens. Adverse events were relatively few, with no associated infections. This retrospective data analysis suggests that further studies are warranted to evaluate ACTH gel in the treatment of nephrotic syndrome.

\section{Acknowledgments}

This report expands upon data presented as a free communication abstract at the 2010 Annual Meeting of the American Society of Nephrology. The authors express their gratitude to MedVal Scientific Information Services, LLC, (Skillman, NJ) for assistance with editing and preparation of tables and figures for the manuscript.

\section{Disclosure}

Drs Bomback, Tumlin, and Gerald Appel have received research support and consulting honoraria from Questcor Pharmaceuticals. Drs Baranski, Bourdeau, Besarab, Alice Appel, and Radhakrishnan have no disclosures. Drs Gerald Appel and Alice Appel are husband and wife.

\section{References}

1. Waldman M, Crew RJ, Valeri A, et al. Adult minimal-change disease: clinical characteristics, treatment, and outcomes. Clin JAm Soc Nephrol. 2007;2:445-453.

2. Palmer SC, Nand K, Strippoli GF. Interventions for minimal change disease in adults with nephrotic syndrome. Cochrane Database Syst Rev. 2008:CD001537.

3. Ponticelli C, Edefonti A, Ghio L, et al. Cyclosporin versus cyclophosphamide for patients with steroid-dependent and frequently relapsing idiopathic nephrotic syndrome: a multicentre randomized controlled trial. Nephrol Dial Transplant. 1993;8:1326-1332.

4. Cattran DC, Appel GB, Hebert LA, et al. Cyclosporine in patients with steroid-resistant membranous nephropathy: a randomized trial. Kidney Int. 2001;59:1484-1490.

5. Cattran DC, Alexopoulos E, Heering P, et al. Cyclosporin in idiopathic glomerular disease associated with the nephrotic syndrome: workshop recommendations. Kidney Int. 2007;72:1429-1447.

6. Segarra A, Praga M, Ramos N, et al. Successful treatment of membranous glomerulonephritis with rituximab in calcineurin inhibitordependent patients. Clin J Am Soc Nephrol. 2009;4:1083-1088.

7. Cattran DC, Wang MM, Appel G, Matalon A, Briggs W. Mycophenolate mofetil in the treatment of focal segmental glomerulosclerosis. Clin Nephrol. 2004;62:405-411.

8. Cattran DC, Appel GB, Hebert LA, et al. A randomized trial of cyclosporine in patients with steroid-resistant focal segmental glomerulosclerosis. North America Nephrotic Syndrome Study Group. Kidney Int. 1999;56:2220-2226.

9. Berg AL, Arnadottir M. ACTH-induced improvement in the nephrotic syndrome in patients with a variety of diagnoses. Nephrol Dial Transplant. 2004;19:1305-1307.

10. Ponticelli C, Passerini P, Salvadori M, et al. A randomized pilot trial comparing methylprednisolone plus a cytotoxic agent versus synthetic adrenocorticotropic hormone in idiopathic membranous nephropathy. Am J Kidney Dis. 2006;47:233-240. 
11. Rauen T, Michaelis A, Floege J, Mertens PR. Case series of idiopathic membranous nephropathy with long-term beneficial effects of ACTH peptide 1-24. Clin Nephrol. 2009;71:637-642.

12. Beck LH Jr, Bonegio RG, Lambeau G, et al. M-type phospholipase A2 receptor as target antigen in idiopathic membranous nephropathy. N Engl J Med. 2009;361:11-21.
13. Lindskog A, Ebefors K, Johansson ME, et al. Melanocortin 1 receptor agonists reduce proteinuria. J Am Soc Nephrol. 2010;21:1290-1298.

\section{Publish your work in this journal}

Drug Design, Development and Therapy is an international, peerreviewed open-access journal that spans the spectrum of drug design and development through to clinical applications. Clinical outcomes, patient safety, and programs for the development and effective, safe, and sustained use of medicines are a feature of the journal, which has also been accepted for indexing on PubMed Central. The manuscript management system is completely online and includes a very quick and fair peer-review system, which is all easy to use. Visit http://www.dovepress.com/testimonials.php to read real quotes from published authors.

Submit your manuscript here: http://www.dovepress.com/drug-design-development-and-therapy-journal 